\title{
SINDACATO E SCUOLA. INTERVISTA A MARTA VIOTTO \\ E FrANCo PILla - SEgRETERIA FlC CGIL VENETO
}

di Nicoletta Masiero*e Alberto Mattei*

Lunedi 23 marzo 2020, in piena emergenza Covid-19, attraverso un confronto via Skype si è svolta la seguente intervista a Marta Viotto, segretaria generale FlcCgil del Veneto (nell'intervista: $\mathrm{MV}$, intervistatori Nicoletta Masiero, $\mathrm{NM}$ e Alberto Mattei, AM). Gli argomenti trattati vertono intorno ad alcune delle problematiche che riguardano l'istruzione primaria e secondaria affrontate anche dai contributi presentati in questo numero di esr dedicato alla scuola. Franco Pilla $(F P)$, referente regionale Flc per la Formazione Professionale, è intervenuto nella parte dedicata.

NM E AM - Innanzitutto chiediamo all'intervistata una breve presentazione per il lettore di economia e società regionale

MV - Io faccio la sindacalista. Provengo dalla segreteria provinciale della Flc Cgil di Treviso. Di professione sono direttore amministrativo presso un Istituto comprensivo di scuola primaria e scuola secondaria di secondo grado. Dopo il periodo in cui ho lavorato per la categoria Flc di Treviso in distacco sindacale, sono stata eletta nel 2016 alla carica di segreteria generale della Flc per il Veneto, ruolo che attualmente ricopro.

NM - Sei arrivata alla segreteria regionale, dunque, ai tempi della cd "Buona Scuola”...

MV - Sì, sono arrivata nel dicembre 2016, in piena riforma della cd. "Buona Scuola". Ho vissuto le peggiori riforme da quando ho avuto

* Ires Veneto, Venezia.

* Dipartimento di Scienze Giuridiche, Università degli Studi di Verona.

DOI: $10.3280 / \mathrm{ES} 2020-001007$

ECONOMIA E SOCIETÀ REGIONALE - ISSN 1827-2479 - XXXVIII(1) 2020 - PROBLEMI E PROSPETTIVE DELLA SCUOLA 
l'incarico come sindacalista. Penso alla riforma Gelmini, nel 2010, fino alla "Buona Scuola", nel 2015, che di buono ha avuto ben poco. Siamo passati da macro-riforme a micro-riforme. Penso ai tagli della riforma Gelmini per la scuola attorno a otto miliardi di euro, con 140.000 persone in meno.

NM - Con tutte queste riforme, in particolare le micro-riforme come le hai chiamate, hai potuto assistere a significativi mutamenti nel processo di reclutamento del personale. Come possiamo oggi, nel 2020, definire questo processo? Sappiamo che in questo senso sono state applicate più logiche, anche confliggenti, come quella concorsuale e quella dell'anzianità meritocratica delle graduatorie. Ma non solo, si è praticata anche la cosiddetta logica definita pratico-abilitante. Come si profila oggi la selezione del corpo docente?

MV - Le micro-riforme hanno prodotto una stratificazione normativa sul reclutamento. Non dimentichiamo che per ogni intervento sulla scuola ci deve essere il visto del Ministero dell'Economia e delle Finanze e, il più delle volte, quest'ultimo ha prodotto delle contrazioni in termini di supplenze. Infatti, la legge di stabilità 190/2014, per il 2015, prevedeva la riduzione della possibilità di effettuare supplenze brevi e saltuarie per il personale docente e A.T.A. aggravando il funzionamento sia della parte didattica che di quella dei servizi amministrativi e ausiliari.

La conoscenza di questo comparto è molto complicata: difficile da governare, complessa da conoscere. Ognuno dei Ministri dell'Istruzione che si sono succeduti ha applicato un'idea di reclutamento diversa. Dalla Scuola di Specializzazione all'Insegnamento Secondario (SSIS) alla Graduatoria Permanente, 1. 124/99, alla Graduatoria a Esaurimento, 1. 296/2006, ai Tfa, Tirocini Formativi Attivi, dm 249/2010, ai Pas, Percorsi Abilitanti Speciali, Ddg 58/2013, ai Fit, Formazione Iniziale Tirocinio, 1.107/2015 dlgs 59/2917, alla legge di bilancio 145/2018 per il 2019 che sostituisce il Fit con il Nuovo Concorso Abilitante e il Percorso annuale formazione iniziale e prova. Questo susseguirsi di norme e provvedimenti governativi ha prodotto un marasma nel reclutamento e come conseguenza una serie molto numerosa di ricorsi ai tribunali ordinari, e amministrativi, con conseguenti difformità di soluzioni e di trattamento. A tal proposito non si può non citare la situazione dei diplomati magistrali ante a.s. 2001/2002.

Abbiamo bisogno di ritornare necessariamente a una cadenza di concorso biennale, che tenga conto del precariato, costituito da un corpo docenti con un'età media già molto elevata. Dovevano essere banditi i concorsi in particolar modo per la scuola secondaria di primo e secondo grado, ma a tutt'oggi non è stato fatto. Mi riferisco sia ai concorsi ordinari che a quelli 
riservati al personale con almeno trentasei mesi di lavoro pregressi, cioè a quel personale docente non abilitato, individuato e nominato dalle graduatorie d'istituto di terza fascia che ha garantito il funzionamento dell'attività scolastica.

NM - Volevamo infatti affrontare la questione dell'elevata età media del corpo docente, la più alta nei Paesi Oecd ...

MV - È un problema. Penso a chi è entrato in ruolo dopo 33 anni di precariato. Se guardiamo le tabelle di Bruno Anastasia (in questo volume, Anastasia: 14-41) vediamo in generale che per il personale in ruolo l'età media è di 54 anni. Comunque, molto elevata anche per il personale a tempo determinato, anche se più bassa: fra i 35 e i 44 anni. Nella scuola primaria si abbassa il numero più alto, perché sono state fatte le immissioni in ruolo, nell'anno scolastico 2017/2018. Abbiamo un corpo docente che è riuscito ad entrare con età elevata. Una laurea, una formazione, un'entrata in graduatoria: sono tutti fattori che spostano in avanti l'età.

NM - Ci sono stati periodi in cui era necessario un test anche per essere ammessi alla formazione abilitante, è corretto?

MV - Sì, per tutta quella tipologia sopraelencata è prevista una serie di test di ammissione, esami e percorsi di durata pluriennale a seconda del percorso di abilitazione frequentato, allungando la fase di entrata in ruolo.

NM - Dal tuo osservatorio, quali sono le conseguenze o alcune conseguenze di questa situazione sul piano professionale e sul piano didattico?

MV - Io credo che l'età non possa essere considerata un limite se c'è esperienza pregressa. Penso, però, al tema della dignità professionale, ad esempio nel confronto fra colleghi, fra posizioni precarie e posizioni a tempo indeterminato. Se ci fosse una formazione continua e permanente, sarebbe una giusta via. Noi abbiamo un problema di non inclusività del personale precario se guardiamo al nostro Contratto collettivo: in termini di diritti, malattia, assenze, si rimane alla prima fascia, con la scadenza al 30 giugno senza retribuzione estiva e in attesa di settembre e ottobre con le nomine, all'inizio dell'anno scolastico. Se poi, invece, la guardiamo sotto l'aspetto educativo, l'età non è sicuramente un limite, se il docente è continuamente formato e partecipa all'attività scolastica come il collega a tempo indeterminato. 
NM - Connessa alla questione della precarietà, con la "Buona Scuola" è esplosa anche la questione della mobilità dei docenti, soprattutto all'avvio della riforma. Oggi come è gestita la condizione dei docenti sotto il profilo dei trasferimenti?

MV - Il problema della mobilità è esploso con la "Buona Scuola", ma ha radici antiche frutto di politiche prive di capacità di investire in maniera omogenea su tutto il territorio nazionale. Faccio un esempio semplice: a Milano il tempo pieno nella scuola primaria interessa più del $90 \%$ delle classi, a Palermo riguarda poco più del $2 \%$. Tempo pieno significa raddoppio dell'orario e naturalmente dell'organico. Naturalmente se noi estendiamo questo esempio alla diffusione della scuola d'infanzia si può capire come la gente si sposti dove il lavoro c'è e poi nel tempo tenti di tornare ai luoghi d'origine. Bisogna anche aggiungere che il blocco quasi ventennale dei concorsi e la scarsità dei corsi di Scienze della Formazione che le università del Nord mettono in campo sono un ulteriore fattore che favorisce questa migrazione.

La "Buona Scuola" aveva previsto un algoritmo. Non siamo riusciti a governare tutta la mobilità del personale, nel senso che ci è capitato di dover gestire chi doveva uscire dalla regione. In Veneto non ci sono stati comunque grandi spostamenti, ma c'è stata attesa.

Sulle nomine, oggi, impatta un organico di diritto che non è organico di fatto. Per effetto del contenimento della spesa pubblica, non avviene regolarmente la copertura di posti in ruolo. Lo stesso algoritmo ha prodotto spostamenti non gestibili. Abbiamo un certo numero di persone per esempio che possono venire in Veneto, ma attendiamo ogni anno l'ordinanza ministeriale. L'occupazione della scuola si rappresenta con molti insegnanti meridionali, ma è una questione storica di scelta da parte di questa Regione.

NM - Un altro aspetto che caratterizza il personale docente è l'alto tasso di femminilizzazione del settore. Una forte presenza femminile tutta da considerare però, se decresce con l'aumento del grado di insegnamento. Anche per quanto riguarda la presenza di donne fra i dirigenti scolastici, c'è stato sì un aumento negli ultimi anni, ma non siamo certo alla parità. Per non parlare della docenza universitaria. Dal sindacato questo aspetto come è valutato e affrontato?

MV - Sicuramente ha un'origine legata al secondo dopoguerra. Il maestro, il prete e il medico erano maschi, prima. Penso alla riforma Gentile. Noi abbiamo una categoria femminilizzata ora, come avviene nella scuola pri- 
maria. Più si eleva il titolo di studio, più si trovano gli uomini e rimane comunque una categoria femminilizzata. Dopo l'unità d'Italia, il maestro era sicuramente maschio, ma ritengo che nel dopoguerra la figura dell'insegnante della scuola primaria è divenuta femminile, sia per l'accesso all'istruzione sia per l'immagine di cura e educazione che contraddistingueva la donna. Tuttavia, lo stipendio rimane basso, poco stimolante per l'ingresso degli insegnanti. Cosa diversa capita per l'Università.

NM - Proprio su questo aspetto, fra i vari indicatori chiave selezionati dalla Commissione Europea (dati riferiti al 2018), colpisce che gli stipendi degli insegnanti italiani sono i più bassi dei Paesi UE, eppure gli insegnanti italiani risultano fra i più soddisfatti. Quale lettura daresti?

MV - Non conosco i dati di questa ricerca né come siano state poste le domande. La mia esperienza alla luce di tante assemblee fatte mi fa dubitare che gli insegnanti italiani siano soddisfatti. Ho percepito una frustrazione sempre più marcata che non riguarda solo l'aspetto economico, ma l'invadenza di procedure sempre più burocratiche che tolgono tempo e voglia al "mestiere di insegnare".

C'è stato uno disconoscimento sociale della figura dell'insegnante che rappresentava un costo sociale invece che un riconoscimento per quanto potrebbe formare i futuri cittadini. Per contro, è la relazione viva ed educativa a compensare a rendere soddisfacente il lavoro in classe. Penso e ritengo che l'insegnante sia nella posizione privilegiata di partecipare alla futura costruzione della società, ogni giorno. Si rinnova ad ogni passaggio di classe, anche con i futuri alunni. Come sindacalista, avverto il peso di questo disconoscimento sociale, credo che l'insegnante non pensi a quanto ammonta il suo stipendio e pensi alla propria dignità, consapevole del proprio ruolo nei confronti di futuri cittadini, a prescindere dal contratto.

$\mathbf{N M}-C^{\prime}$ è un disconoscimento sociale che si traduce in un mancato riconoscimento sul piano retributivo, dovuto anche al generale contenimento della spesa, ma per alcuni insegnanti sembra esserci anche il serio pericolo di un disconoscimento del ruolo dal punto di vista formale. Pensando, per esempio, all'Invalsi, al Pisa, quale può essere l'impatto di strumenti di questo genere? L'applicazione, anzi l'obbligatorietà, di questi dispositivi di valutazione rischia di provocare una loro sovrapposizione al ruolo dell'insegnante, declassandolo? Puoi dirci come è vissuto questo possibile rischio, se avvertito? 
MV - Credo che nessuno sia contrario in maniera preconcetta al sistema di valutazione scolastica. Qualche criticità c'è stata, penso ad un territorio disomogeneo come quello nazionale. Anche all'interno di un medesimo territorio regionale dove ci sono più disagi sociali in una zona rispetto ad un'altra, una prova standardizzata corre dei rischi. Il problema che ho visto è che l'Invalsi non è tornata utile, anzi è servita per disconoscere il lavoro di questo e quel docente o per mettere una scuola a confronto con un'altra. Il sistema di valutazione potrebbe essere utile se ci fosse un'autovalutazione da parte dello stesso corpo docente. Dico questo perché l'Invalsi è stato imposto dall'alto, senza coinvolgere la comunità scolastica. Di fatto, si è generata un'ansia da prestazione, creando un clima di competizione e non di cooperazione. I dati, se non mediati e non guidati, producono fratture all'interno della comunità stessa.

NM - Riprendendo questa disomogeneità territoriale di cui hai detto, oltre alle criticità legate alla professionalità dei docenti, l'altra questione che emerge sempre, quando si parla di Invalsi, riguarda la sua reale efficacia nella lotta alle diseguaglianze. Forme di valutazione di questo tipo si basano sull'idea che il "merito" rappresenti la soluzione alle disfunzioni del sistema e vengono presentate come misurazioni "oggettive" delle capacità acquisite, del valore dell 'istruzione impartita e quindi in grado di contribuire "scientificamente" all'eliminazione delle disuguaglianze riconoscendo il merito e l'eccellenza. Ma altre narrazioni, proprio per questo, vedono nell'Invalsi il grimaldello per perpetuare le disuguaglianze. Qual è la vostra riflessione su questi nodi?

MV - Siamo entrati nella logica della misurazione di tutto. Se è misurato, è scientifico e attendibile. Mi chiedo: dove si sono avuti risultati disastrosi dell'Invalsi si è avuto poi un intervento? A me non risulta. Non è servito per migliorare la qualità della scuola. Che cosa vogliamo misurare? E una volta misurato, cosa vogliamo fare? Preciso che l'Invalsi non ha comunque un valore didattico, in quanto estromesso dal curriculum dello studente per valutarlo in sede di esame di Stato, ma serve comunque per accedere all'esame della maturità, come requisito di accesso.

Dobbiamo prestare molta attenzione: puntare su prove standardizzate rischia di curvare anche la didattica su logiche standardizzate. L'editoria sforna centinaia di libri che simulano prove Invalsi e quindi il rischio è quello dell'addestramento in funzione della riuscita nella prova. Se poi dovesse passare l'assunto che buona prova uguale bravo insegnante magari con ricadute contrattuali allora saremmo proprio nei guai. 
NM - Per restare al tema delle diseguaglianze, uno dei contributi presentati in questo numero di esr esamina le difficoltà con cui gli studenti stranieri (in questo volume Tosi, Borgna, Belloni: 42-59), a parità di titolo di studio, si scontrano nel mercato del lavoro. L'istruzione "non paga" nel superare le disuguaglianze. Ma prima di questo, prima dell'accesso al lavoro, qual è la situazione degli studenti stranieri in una regione come il Veneto? Che cosa ci puoi dire a riguardo?

MV - Noi contiamo 93.074 studenti, in tutti i gradi di scuola, di cittadinanza non italiana nel Veneto. La provincia che ne ha di più è Verona, quella che ne ha meno è Belluno, per ragioni geografiche e lavorative. Il numero di alunni stranieri a metà degli anni Novanta era contenuto (circa 50.000 ), ora i numeri sono molto alti (circa 800.000). Allora c'era una generazione sospesa, non la seconda generazione che c'è oggi e che non ha vissuto quella fase di sradicamento, ma oggi questi studenti vivono una discontinuità culturale, con regole e valori contrastanti dentro la famiglia stessa. Questo è un aspetto molto delicato, perché l'alunno diventa il mediatore culturale dentro la famiglia legata alla terra di origine. In questo scenario, la scuola ha giocato un ruolo fondamentale, seppur in maniera disomogenea, mettendo in crisi l'identità nazionale di provenienza e mettendo in evidenza l'arretratezza della nostra normativa sulla cittadinanza. Se avevamo come faro la Dichiarazione dei diritti dell'uomo nel 1948, poi la Convenzione sui diritti dell'infanzia del 1989, dichiarazione che l'Italia ha fatto propria, la scuola si è trovata in solitudine, cercando la via dell'interculturalità. La scuola è stata la prima "agenzia" ad intervenire a far fronte a questo problema. C'era la necessità di un riconoscimento della diversità, ma oggi è altrettanto importante fare attenzione all'apertura a queste diversità. Penso al concetto dell'interculturalità che è stato introdotto da una quindicina di anni: dialogo trasversale tra culture mette in evidenza che le diversità sono una ricchezza, senza che ci sia un approccio etnocentrico (circolare n. 205 del 1990). La via italiana alla scuola interculturale è stata scritta nel 2006 e la scuola, in questo senso, era preparata. C'era questo orizzonte. La politica ha fatto invece credere cose diverse: politica di diffidenza e di paura, senza bisogno di fare degli esempi. La scuola come "agenzia", ma al di fuori di tale perimetro, la cittadinanza non era pronta ad accogliere e governare. Oggi il tema è lo ius soli, da non vivere come un premio di assimilazione, che però non viene affrontato in quanto passaggio, da vivere all'interno della società.

NM - Il tema sembra accantonato dall'agenda politica ... 
MV - Non trova consenso politico, oggi.

NM - Uno dei contributi) che presentiamo in questo volume (D'Agostino, Vaccaro: 89-104) è dedicato al cosiddetto sistema duale nell'Istruzione Professionale, che per certi versi è un capitolo a sé, di competenza della Regione. Puoi tracciare, Franco, un quadro di questo comparto?

FP - Il sistema educativo della Regione Veneto si articola secondo la legge n. 8/2017. La nuova legge regionale trasforma i Centri di Formazione Professionale in Scuole di Formazione Professionale, in continuità con la riforma voluta dalla ex ministra della P.I. Letizia Moratti con la legge 53/2003, che aveva previsto l'assolvimento dell'Obbligo Scolastico anche nei percorsi della Formazione Professionale.

Il sistema di Istruzione e Formazione Professionale (IeFP) è scelto da circa 20 mila studenti veneti, ossia il 13\% della popolazione scolastica regionale delle scuole superiori. In Veneto operano 80 Scuole di Formazione Professionale, che erogano circa 900 corsi suddivisi in 21 percorsi di qualifica di durata triennale e 49 percorsi di diploma di quarto anno riconosciuti a livello nazionale. I corsi del quarto anno di diploma sono svolti secondo il sistema "duale", che comprende 500 ore di formazione a scuola e 500 ore in azienda.

Gli Enti di Formazione accreditati dalla Regione Veneto all'assolvimento dell'Obbligo Formativo sono 108. Il soggetto che rappresenta il numero più consistente di Enti di Formazione accreditati è Forma Veneto, presente in 44 comuni del Veneto con 73 Scuole di Formazione Professionale, che nell'anno scolastico 2017/18 hanno attivato 893 corsi coinvolgendo 17.464 studenti, che rappresentano 1'86\% della totalità (20.307) di allievi inseriti nei corsi IeFP nella Regione Veneto. Oltre al sistema della IeFP ci sono 11 Centri che operano nell'handicap grave.

AM - La situazione del personale ha delle peculiarità anche in termini contrattuali?

FP - I dipendenti nel sistema di IeFP sono circa 2.000, ai quali vanno aggiunti circa altri 1.800/2.000 lavoratori con rapporto di lavoro co.co.co. e partita Iva. Ai lavoratori che operano all'interno dei bandi dell'Obbligo Formativo viene applicato obbligatoriamente il Ccnl per la Formazione Professionale. Il Ccnl prevede altri due livelli di contrattazione regionale e di Ente. Al tavolo della contrattazione regionale i soggetti sono gli Enti "Forma Veneto" e le OO. SS. Regionali, mentre al tavolo della contrattazione di singolo Ente si vedono l'Ente e le RSU. In Veneto, la contratta- 
zione regionale ha chiuso accordi riguardanti diverse materie, che vanno dall'organizzazione del lavoro, alla flessibilità per i formatori, dalle missioni, ai premi incentivanti, dal diritto all'aggiornamento/studio, al welfare, alla sanità integrativa. Inoltre, si è arrivati alla costituzione dell'Ente Bilaterale EBiRFOP che ha erogato fondi per integrazione al reddito nelle situazioni di cassa integrazione in deroga, contributi in caso di licenziamenti per esubero del personale, contributi per la sanità integrativa.

AM - Sul piano pedagogico formativo in questo particolare comparto ci sono criticità?

FP - Il sistema della IeFP raggiunge circa il $90 \%$ del successo formativo negli esiti occupazionali. Circa il 35\% degli allievi continuano un percorso formativo, il 55\% trova lavoro entro un anno, e solo il 10\% rimane non occupato. Inoltre, secondo il Miur, in Veneto la dispersione scolastica è all'8,7\% I percorsi di istruzione e formazione professionale in Veneto recuperano circa 4.000 allievi "espulsi" dal sistema di istruzione. Questi dati hanno un impatto notevole sulla dispersione scolastica, che la Strategia di Lisbona fissa ad un massimo del 10,5\%.

NM - Parliamo dell'Alternanza Scuola-Lavoro. Dopo i problemi iniziali, il Pcto ha portato a una macroscopica riduzione delle ore obbligatorie. La Flc però ne ha chiesto il ritiro, sia per ragioni contrattuali, per es. sovraccarico del lavoro per tutto il personale scolastico, sia per ragioni formative, sostenendo che $i$ nuovi percorsi farebbero ruotare la didattica attorno all'imprenditorialità e all'economia, rendendo protagonista l'azienda, mentre sono gli esiti delle attività del Pcto che devono avere una ricaduta sugli apprendimenti disciplinari e non il contrario. Che cosa è davvero cambiato rispetto all'Alternanza oltre alla riduzione delle ore?

MV - Agli inizi degli anni Duemila l'Alternanza Scuola-Lavoro entra a pieno titolo nelle scuole. Nasce come metodologia didattica, in cui la responsabilità rimane in capo alla scuola per assicurare agli studenti, che hanno acquisito delle conoscenze di base, compresa anche l'acquisizione di competenze spendibili nel mercato del lavoro. Il percorso doveva essere formativo e coerentemente co-progettato con l'azienda, da valutare e documentare. L'attuale Percorsi per le competenze trasversali e per l'orientamento (Pcto) dovrebbe andare a migliorare i difetti dell'Alternanza Scuola-Lavoro: 1'obiettivo è acquisire attitudini per il proseguimento del percorso di studio. Le competenze sono trasversali, o almeno dovrebbero esserlo. Il problema è da come siamo partiti: c'è un pro- 
blema di come declinare la formazione all'interno delle aziende stesse, senza che ci sia una cultura educativa e formativa attraverso cui si arriva agli studenti. A tutt'oggi gli studenti svolgono mansioni non adeguate, nonostante si siano fatti dei miglioramenti (es.: scelta di alcune aziende rispetto ad altre).

NM - Se capisco bene, dici che l'obiettivo è acquisire attitudini per il proseguimento del percorso di studio e quindi non solo per il lavoro. Intendi dire che l'orientamento non è esclusivamente verso il mercato del lavoro? $\grave{E}$ in questo che consiste lo spirito del nuovo Pcto, non in una semplice riduzione delle ore obbligatorie come sostengono alcuni?

MV - Dovrebbe essere nello spirito del Pcto. Quando si parla di formazione non si può parlare di cultura del lavoro, ma di cultura che viene portata nel mondo del lavoro, anche per l'accrescimento dell'azienda stessa, come interscambio virtuoso. Dobbiamo fare comunque i conti con il rischio opposto, ossia della aziendalizzazione della scuola, considerato comunque il contesto veneto di piccole-medie imprese. La Pcto è l'Alternanza ScuolaLavoro ad orario ridotto. Quel mondo del lavoro non è in grado di accogliere questo scambio con l'istituzione scolastica. Sarebbe importante una co-progettazione, bisognerebbe personalizzare il percorso di studi all'interno dell'azienda stessa.

NM - I due mondi dovrebbero rimanere distinti, senza strumentalizzare l'istruzione alle esigenze delle imprese, del mercato ... Eppure, nonostante tutte le difficoltà incontrate e denunciate, pare che gli studenti, nella maggior parte dei casi, apprezzino l'opportunità di fare esperienza in azienda. Che cos'è non capiamo?

MV - Se vissuta dal punto di vista umano, può avere dei risvolti positivi. Altra cosa è pensare che quel tipo di esperienza possa avere un valore formativo, che in un mercato del lavoro addestrato ci possa essere quell'aspetto innovativo. Non basta saper fare una cosa per insegnarla. È necessario un passaggio dell'educazione, anche per accrescere.

AM - Autonomia scolastica e autonomia regionale differenziata, di cui si è molto discusso in Veneto: quale valutazione ne dai?

MV - Il sindacato è stato sempre molto contrario. Penso al Referendum del Veneto, che ha spinto in direzione di un identitarismo marcato. C'è stata un'ampia partecipazione al voto, pari al 57,2\% degli aventi diritto, con una 
maggioranza di sì (98\%). In quel periodo era sicuramente uno slogan utilizzato, immaginandosi la Regione più efficace e più efficiente del Ministero, a livello nazionale. Il Referendum proponeva quel quesito referendario chiedendo di normare regionalmente le 23 materie, tra cui l'istruzione. $\mathrm{Su}$ questo abbiamo espresso la nostra posizione contraria, perché riteniamo lo Stato garante della solidarietà e con un ruolo primario. Capiamo la diversificazione che c'è e come si rappresenta. Ne conseguirebbe una diversificazione nella concreta esigibilità di alcuni diritti che pensiamo fondamentali.

\section{AM - Per esempio?}

MV - Penso al Contratto collettivo nazionale e all'idea che il Contratto collettivo verrebbe regionalizzato, anche dal punto di vista economico. Quello che non si capisce è quali siano la vera motivazione e la vera finalità di questa operazione. Se per l'università c'è già autonomia, nella scuola abbiamo come riferimento il Contratto nazionale. Ritengo che i principi costituzionali, come il principio di uguaglianza dell'art. 3 della Costituzione, ma anche l'art. 33 sulla libertà di insegnamento e l'art. 34 sul ruolo della scuola per "i capaci e i meritevoli", sarebbero a rischio e con l'autonomia differenziata ci sarebbe un aumento del divario tra Nord e Sud, ma anche tra i territori stessi del Veneto. Ecco perché penso che lo Stato debba essere garante in questo senso, altrimenti si rischia la frantumazione della cultura stessa del Paese.

NM - Potrebbe cadere la validità del Contratto nazionale, passando ad una contrattazione territoriale, diciamo di secondo livello? E le rappresentanze sindacali che ruolo dovrebbero allora svolgere?

MV - Il ruolo non muta. Oggi non c'è una disparità, perché si guarda all'istituzione scolastica. Se ci fosse un cambiamento nell'assunzione si avrebbe una regionalizzazione, ma non è chiaro ad oggi l'aspetto economico. Si fa riferimento ad un'indennità del personale dipendente della Regione Veneto. Così si avrebbe diversificazione e si finirebbe col perdere il ruolo che riveste oggi il Contratto collettivo nazionale. Dovremmo, invece, sempre di più, disporci al fatto che gli studenti si sentono studenti europei e dovrebbero essere orientati ad una logica indirizzata all'Europa.

Io credo che uno Stato come l'Italia dovrebbe applicare 1'art. 119 della propria Costituzione, tentando di rimuovere gli squilibri economici e sociali per favorire il libero esercizio dei diritti della persona. Tenendo fede anche all'art. 120 della Costituzione, secondo cui ci dovrebbe essere 
l'intervento sostitutivo dello Stato dove alcune Regioni lo richiedono. Penso, in questo senso, all'edilizia scolastica.

NM - Dal punto di vista delle relazioni sindacali quindi ci sarebbero delle conseguenze ...

MV - L'attuale proposta della Regione Veneto sull'autonomia differenziata può modificare radicalmente l'assetto istituzionale delle relazioni sindacali. Questo progetto fa un uso strumentale della Costituzione attraverso un'interpretazione capziosa degli articoli 116 e 117 . Il progetto tende ad enfatizzare le differenze fra i territori del nostro paese, realizzando, attraverso l'utilizzo del residuo fiscale, una revisione dell'intero comparto dell'istruzione.

L'evoluzione del quadro politico non ci consente al momento di fotografare una situazione definita e di conseguenza pensare a politiche sindacali regionali che siano in grado di garantire insieme ai diritti per i lavoratori che rappresentiamo anche la garanzia di un servizio per l'utenza basato su criteri di qualità, dalla scuola dell'infanzia fino all'università, in una logica non egoistica di solidarietà nazionale. Se dovesse passare il disegno della Regione Veneto, che prevede il trasferimento alla regione di una serie imponente di materie, dalle finalità del sistema alla disciplina del rapporto di lavoro del personale della scuola e degli uffici, dalla programmazione dell'offerta formativa integrata ai contributi alle istituzioni scolastiche paritarie e molto altro, è ovvio che l'attuale impianto delle politiche sindacali dovrebbe cambiare radicalmente, partendo dalla struttura stessa dell'organizzazione sindacale.

NM - E per quanto riguarda le politiche del personale, cosa accadrebbe?

MV - I dirigenti scolastici da subito diventerebbero dipendenti regionali. Partire dai dirigenti non è certo una scelta casuale, perché è evidente che in questa situazione i dirigenti sarebbero molto più dipendenti dal decisore politico, con i conseguenti condizionamenti sugli orientamenti da dare al sistema formativo.

Anche i nuovi assunti e il personale precario sarebbero da subito dipendenti regionali e anche in questo caso il condizionamento è più che evidente. Tutti gli altri dovrebbero decidere se restare dipendenti statali o optare per il sistema regionale. È evidente che questa possibilità di scelta è del tutto teorica, perché è impossibile governare un sistema dove persone che svolgono lo stesso lavoro o hanno la stessa mansione possano avere poi dei datori di lavoro diversi e norme diverse che regolano il loro status. 
È quindi fuori dubbio che le politiche sul personale condizioneranno anche il ruolo del sindacato, che si dovrà muovere in una dimensione inedita, tra un contratto nazionale residuale e un contratto integrativo regionale che sarà il nuovo vero contratto. E questo attacco al contratto nazionale potrà avere conseguenze bene al di là del personale della scuola. Uno degli elementi che alcuni media hanno enfatizzato è il confronto con le province autonome di Trento e Bolzano e il relativo vantaggio economico che ne deriverebbe ai lavoratori della scuola. Un'attenta lettura dei contratti di queste province evidenzia che l'aumento salariale è reale, ma tutto legato all'aumento dei carichi di lavoro, sia per l'attività di docenza che per quella di formazione e anche per la sostituzione dei colleghi assenti. Bisogna anche ricordare che i contratti integrativi di queste province non alterano lo status giuridico del personale docente, che resta sempre un dipendente statale.

AM - Nella Bozza della Regione Veneto c'è anche la parificazione del sistema pubblico con quello privato....

MV - Questo elemento, da sempre bandiera della destra politica, di fatto più che essere un elemento perequativo del sistema pubblico rischia di limitare fortemente la sua qualità e le garanzie e i diritti del personale. Già ora la Regione Veneto ha competenze in materie di formazione professionale e tutti i dati ci dicono che è aumentata la precarizzazione del personale e si sono ridotti gli investimenti, tanto che molti centri di formazione professionale hanno difficoltà a proseguire con la loro attività.

Un altro tema che è presente nella bozza e che tocca l'essenza stessa del processo formativo è il rapporto tra il sapere e il saper fare, tra scuola e lavoro. La logica che sottende alle politiche regionali in merito all'Alternanza Scuola-Lavoro, ora Pcto, è una logica curvata sul mondo delle imprese, come si diceva prima. È e sarà compito della Flc ribadire che sapere e saper fare sono essenziali nella formazione delle persone, ma solo se le scuole sono messe nella condizione di stabilire la durata dei percorsi in piena autonomia, individuando in maniera rigorosa la qualità formativa delle imprese e scartando quelle che non si preoccupano dei diritti dei propri lavoratori.

AM - Prima è stata evocata una questione spinosa, quella della situazione degli edifici scolastici. A questo proposito ci puoi dire che implicazioni ha questo tema per la platea di persone che sono presenti nella scuola, dagli insegnanti agli studenti? A volte si ha l'impressione che sia un tema sottovalutato che emerge solo quando c'è una reale emergenza. 
MV - L'edilizia scolastica nel nostro Paese rappresenta una vera e propria emergenza nazionale ma lo stato e la qualità degli edifici scolastici rappresentano un territorio e suoi abitanti. Diventano indicatori di quanto una comunità investa nella sicurezza e la formazione dei cittadini più giovani. Il benessere è anche luogo dove si avvia un percorso in cui la relazione di un gruppo di persone diverse è anche condivisione e negoziazione per la produzione di idee e ci consente di fare dei progetti originali, dove la creatività di ognuno si possa esprimere.

A testimonianza della estrema vicinanza tra pensiero filosofico e architettura, in una celebre conferenza del 1951 di Martin Heidegger, "Costruire abitare pensare": «L'essenza del costruire è il "far abitare". Il tratto essenziale del costruire è l'edificare luoghi mediante il disporre i loro spazi. Solo se abbiamo la capacità di abitare, possiamo costruire»1. Occorre infatti, superare l'approccio di intervenire prevalentemente sui casi di emergenza per arrivare a una programmazione degli interventi e della manutenzione ordinaria e straordinaria, prevedendo anche un piano di riqualificazione per la messa in sicurezza, la bonifica e la sostenibilità degli edifici. Questa operazione di riqualificazione consentirebbe di considerare l'edilizia scolastica una delle grandi opere pubbliche nazionali, che darebbe, oltretutto in tempi di crisi economica, occupazione e miglioramento territoriale di un patrimonio edilizio diffuso in maniera capillare in tutto il Paese.

Mai come da questo periodo segnato dalla pandemia del coronavirus il problema dell'adeguatezza degli spazi scolastici diventerà fondamentale per una ripresa a settembre delle lezioni. Nel Veneto la maggior parte degli edifici scolastici risale agli anni Sessanta-Settanta, quando c'era bisogno di rispondere al boom della scuola di massa. Stiamo parlando di edifici che hanno più di 50 anni, strutturati con criteri per cui l'aula era il centro dell'edificio. Ora servirebbero spazi multimediali adeguati ai nuovi bisogni specialmente se la didattica a distanza diventerà anche una parte della didattica in presenza. Temo che non ci saranno risorse per fare questa rivoluzione copernicana. Temo che settembre sarà drammatico perché ci saranno doppi turni. Riduzioni del tempo scuola e centinaia di migliaia di precari. Spero molto di sbagliarmi.

1 Conferenza tenuta il 5 agosto 1951 nel quadro del Secondo Colloquio di Darmstadt su Uomo e spazio. Oggi in Heidegger M. (2014), cura e trad. it. G. Vattimo. Saggi e Discorsi, p. 107. Milano: Mursia pp. 96-108 (ndr). 